\section{CLINICAL LECTURE ON}

\section{THE HOS PITA PLAGUES.}

By JONATHAN HUTCHINSON, F.R.C.S.,

Senior Surgeon to the London Hospital, and to the Hospital for Skin-Diseases.

Gentlemen, - I wish to give you in the present lecture a sort of bird'seye view of certain diseases which are apt to occur with especial severity and more than usual frequency in hospital practice, and which have been aptly termed the plagues of the hospital surgeon. I allude to those which are known under the names of erysipelas, pyæmia, septicæmia, and hospital gangrene. We have on many former occasions discussed each of these maladies separately. I propose now to give you a sort of recapitulation of the remarks which, in a more diffuse manner, have been many times repeated at the bedside. In beginning, let me say that it is hopeless to get clear ideas on this rather complicated subject, unless we carefully define the terms that we use. I must beg you to try to keep in mind throughout the lecture the special explanations of terms which I shall give.

We will take first the commonest, and for that reason the most important, of the diseases in question, Erysipelas. By this term, we designate a peculiar form of inflammation which shows a remarkable tendency to spread over large surfaces, subsiding where it commenced, and advancing to adjacent parts. It is characterised by free fluid effusion producing soft œdema, by paralysis of the blood-vessels producing vivid redness, and by fever disturbance or pyrexia, which is proportionate to the extent of skin involved. In connection with the tendency to fluid effusion, vesications are frequently seen under the epidermis, and pitting on pressure is always noticed. The area of inflamed skin with capillary paralysis is usually abruptly defined, and a well-marked margin is consequently a very characteristic symptom in most cases. A typical example of erysipelas (or, perhaps, we had better say, of erysipelatous inflammation of the skin) may be recognised at a glance by the vivid redness, the spreading edge, the vesications, and the puffy swelling. There may be minor degrees of erysipelas in which the characters are ill marked, there may be peculiar forms in which one or more of the usual symptoms are absent, but, for the present, I will speak only of what is the rule. Now, I am anxious that you should understand clearly my position, when I assert that erysipelas is simply a peculiar type of the inflammatory process, and not a specific disease. The distinction is of the utmost importance. A specific disease is one which can be bred only by the implantation of its seeds. Small-pox, measles, and syphilis are specific diseases; and they can be produced, as everyone knows, only by the introduction of their specific germs. You may know a specific disease by its having a special course and observing definite stages. It takes time to develope, the incubative stage; then has a stage of outbreak, usually attended by a skin-rash, the exanthem or efflorescence stage ; and, lastly, has, after lasting for a definite period, its stage of steady decline. Unless you interfere with its evolution by treatment, these stages will be, within certain limits, of the same average duration in all persons. That a specific disease is a blood-malady, will be proved by the symmetry with which its manifestations are produced; and, lastly, by the fact that the patient's body remains for a longer or shorter period, but often for life, insusceptibile of a repetition of the phenomena. Now, keeping these tests in mind, everyone must acknowledge, after five minutes' thought, that erysipelas is not a specific fever. It observes no definite stages, can be produced de novo, is never symmetrical, except by accident, and, so far from protecting the patient's system, leaves him more susceptible than before. In the exanthemata, the skin-eruption is produced by the blood-disease, and does not appear until after the febrile disturbance has set in ; whilst in erysipelas the blood-changes are secondary to the skin-inflammation, and the pyrexia does not precede the local disease. To group erysipelas with the exanthemata, simply because it is attended by redness of the skin, is to take a jump in classification little less bold than that ventured by the man who, seeing an ass for the first time, declared that he kad "now found the father of all the rabbits." We must not be guided by length of ears alone, but must be content in a painstaking manner to estimate all the features of peculiarity which are presented for our consideration. I most sincerely hope that our systematic works, most of which still speak of erysipelas as a specific fever, will desist from doing $\mathrm{s} n$, or else justify the term. It is no question of mere words; for our notions as to the cause, the method of prevention, and modes of treatment, will depend upon whether or not my assertion is correct.

You will meet with erysipelas in our wards chiefly under two forms - what is called idiopathic or medical erysipelas, and surgical or traumatic erysipelas. The two are in most cases very different ; the latter being a very serious, and the former a comparatively mild disease. Idiopathic erysipelas almost always begins on the head or face, and rarely spreads lower than the shoulders, and it often occurs over and over again in the same patient, each attack being milder than its predecessors. For the most part, it is first attacks only which are attended by any danger to life. As regards the causation of idiopathic erysipelas, one fact is certain, and from it, I think, we may venture to infer a good deal. It is undoubtedly very often caused by exposure to draughts. In almost all first cases, this influence as an exciting cause has been recognised, and those who are liable to recurrent attacks usually know well what to avoid. It is rare, indeed, to find anyone who suspects stomach-disturbance or any other derangement of general health as the cause. It may occur to those who are apparently in perfect health, and, indeed, all the facts with which I am acquainted justify the conclusion that it is a local inflammation produced by a local cause. If the evidence in this direction is strong in the case of medical erysipelas, it is yet more so as regards the traumatic form, for here we have existence of a definite local lesion. Now, although traumatic erysipelas is in undoubted connection with and dependence upon the existence of wounds, yet it is not specially associated with severe wounds. The most trivial break of surface may suffice, if certain other conditions are added. What are those conditions? The following may, I think, be enumerated :- I, exposure of the unhealed wound to cold : let a patient with a scalp-wound and a bald head sleep with an open window above, ten to one but the result will be erysipelas-this is what we have just asserted to be true of the idiopathic form, which follows exposure to cold air, especially if it take place during sleep; 2 , excessive irritation of the wound-thus it is highly probable that tight stitches are occasional causes, and that wounds attended by contusion or laceration, such, for instance, as saw-cuts, are more liable to erysipelas than others ; 3 , the introduction of irritants, and, above all, of the secretions of unhealthy wounds. This involves the doctrine of contagion; and respecting it, I hold strongly that there cannot be the least doubt that the secretions of erysipelas are capable of conveying the disease from patient to patient. All living pus, indeed all living secretions produced by inflamed parts, are probably capable of producing, when transferred under favourable circumstances and to appropriate soils, inflammations similar to those from which they had their origin. The truth of this we see exemplified every day in the case of gonorrhœa, gonorrhœal ophthalmia, purulent ophthalmia, catarrhal ophthalmia, and many other diseases. Its assertion involves the idea of specificity only in a very minor degree ; and, at any rate, it is but a specific local disease, not a blood-malady, which is the product. In this sense, we assert the contagiousness of erysipelas. It is probably very contagious, that is, a very small quantity of the materies may suffice, and it is by no means certain that it may not sometimes spread through short distances by aërial infection. The frequency with which a patient in a bed adjacent to one suffering from erysipelas contracts it, is familiar to all surgeons. The cases, however, in which surgeons engaged in dressing erysipelas have been the means of producing metritis and puerperal fever in their midwifery patients, are amongst the most instructive. In these, we are obliged to believe that the contagion may cling about the hands of the surgeon for days together, and in spite of repeated washing, or possibly that his coat-cuffs may be its domicile. Surely, we cannot hesitate to admit that what has been so well proved in the case of the accoucheur may be possible also to the nurse, the dresser, or the operating surgeon, and that the hands of all these may, without any unusual degree of carelessness, become the means of conveying contagion.

Guided by the considerations just stated, we seem to have clear in dications for the prevention of erysipelas in hospital practice. We have first to prevent its autogenetic production by placing all wounds under favourable conditions as regards freedom from irritation, and, above all, not risking any exposure to draughts of cold air; secondly, if, in spite of all precaution, cases have arisen in our wards, or if we have been compelled to admit them from without, it is our duty to guard against the contagious spread of the disease by most carefully isolating all its subjects. No one who has anything to do with healthy wounds ought to go near erysipelas cases.

If you ask me whether erysipelatous inflammation is productive of blood-poisoning, I answer, No, not as a rule. It causes over-heating of the blood and it irritates the nervous system, and is thus productive of the usual phenomena of pyrexia; but these are always proportionate to the extent of the local disease, and they subside with its subsidence. There is not, as a rule, any coagulation of the blood in the veins, and 
thus no risk of blood-poisoning in the worse sense of that term. Most erysipelas patients recover. If death occur, it is usually due to great extent of disease, to the importance of the part implicated, or to unusual severity in the type of the inflammation, causing gangrene, etc. In a few cases, however, we must admit that symptoms of what is called septicæmia are developed, and in a few others those of pyæmia.

We will now pass to the consideration of these latter maladies. In reference to Pyxmia, I believe you will find all authorities agreed that, when a patient suffers during life from repeated rigors, and presents after death numerous abscesses in the viscera, this term is applicable. These two things generally go together; when rigors are numerous and well marked, internal abscesses will scarcely ever be wanting. There are, however, a great number of cases which are called pyæmia in which these conditions are ill marked, and some in which it is extremely difficult to say whether the term ought to be used or not. The decision will rest upon the occurrence or otherwise of abscesses. In proportion to the proved multiplicity of the suppuration, will the pyæmic nature of the malady be established. We must ask next what is the cause of the abscesses; and to this the reply is, that they result from the embolic arrest of fragments of material detached from primary phlebitic clots. This material is not of the nature of an inorganic or even merely fibrinous plug; but, owing either to its being living, or to its being in process of decomposition, it is capable of setting up suppurative action at the site of its arrest. We have now, I think, advanced a very definite step in our conception of what pyæmia is. There is no pyxmia unless fragments are in circulation capable of acling, when detained, as inducers of inflammation, and these fragments in turn can be derived only from the interior of diseased veins. The phenomena of pyæmia have their origin in disease of the veins. We will not stop to ask whether the lining membrane of veins can suppurate or not ; it is sufficient for our argument that all admit that the veins can inflame, and that, as one of the results of their inflammation, clots or concrete masses may form within them, which, in turn, may break up and furnish emboli of the most dangerous kind. We admit most fully that it is absolutely impossible to distinguish a pus-cell from a white blocd-corpuscle; that, indeed, they are probably identical organisms; and that thus no one can possibly prove the presence of pus in the blood. This is a mere matter of detail. We know quite enough to admit of our defining pyæmia as the disease produced by the circulation of embola derived from the interior of an inflamed vein. It would, perhaps, be better to call the malady phlebitic blood-poisoning; but the name "pyæmia" is in possession, is fairly well understood, and will probably keep its place, with the conventional limitation of its meaning just suggested.

Now, in hospital, we meet with some splendidly definite examples of phlebitic blood-poisoning (=pyæmia). They occur in connecticn with contusions of bone, which have been followed by osteitis and death, and are especially common after severe lacerations of the scalp. They occur, also, where bones have been sawn across in amputations, or broken in compound fractures. They are met with also after operations on veins, ligature of piles, phlebotomy, tying of varicose veins, and the like. Lastly, we meet with them occasionally in connection with carbuncles and other unhealthy forms of skin-inflammation. They are rare after operations which do not involve either veins or bone. Now, the reason why bones and veins are classed together in this association is easily given. In the cancelli of bones are large venous channels, and when the surrounding bone is in process of death, there is great risk that these channels may become diseased, and that plugs may form within them. The slowness of the process of death in bone favours this result, for the more rapid the death of an inflamed part, and the separation of its structure from the living, the less the danger of absorption of its fluids. In the most characteristic cases of pyæmia, the patient often appears to be going on very well until the day of the first rigor ; and this rigor, in all probability, denotes the formation of the first abscess. Thus the inflammation of the bone and vein, or of the vein only, which was to cause the patient's death, may have been latent, and the first symptom to excite alarm may be one proving that blood-contamin ation has taken place, and that embola, derived from an inflamed vein, are in circulation. It is by no means a matter of mere chance as to where these embola stick fast. They will probably, if large, be filtered out by the first capillary which they encounter ; but if they have once passed through the lungs and heart and gained the systemic arteries, they may then be expected to stop wherever the capillaries are smallest. If there be any part which has been injured, and where already, as the result of contusion or fracture, some blood-vessels are stopped, there very probably an embolon may be arrested and do its work. The capillaries of the lungs and liver are very likely to stop them, and so also the small vessels in proximity with the cartilages of the joints.

Now, as regards the autogenetic production of pyæmia, no doubt can be felt that most of the examples of it which we see are of this kind. We admit into hospital many of the worst cases with pyæmic symptoms already well marked. Young children with acute periostitis (osteitis) of the long or flat bones, and men with laceration of the scalp and contusion of the skull, who have not thought themselves sufficiently ill to seek admission until the first rigor occurred, such are our usual subjects. On the other hand, although I firmly believe that many pyæmic cases originate in hospitals in connection with contagion, yet it is much more difficult to prove this than in the case of erysipelas. Many of the cases beginning in hospitals are also probably in no way connected with hospital influ. ences, and would have occurred just the same had the patient been treated at home. Still I repeat, whatever causes a wound, in connection with a broken or cut bone or which involves veins, to become unhealthy, increases the risk that pyæmia may result. Not improbably it, like erysipelas, may be produced by contagion from the surgeon's hands or instruments.

In reference, therefore, to the prevention of pyæmia, it may be as. serted that the majority of cases are not susceptible of prevention in any way, whilst certain others may be prevented by precisely the same measures hinted at in the case of erysipelas. Nothing can be more ab. surd than to speak of pyæmia as if it were a disease of hospitals only, for it occurs in private houses, and is, indeed, common in the lower animals $(e . g$. sheep and lambs) treated in the open field. If, however, pyæmia be found to be frequent in a hospital, if it occur after operations, then at once a suspicion should be aroused that it is not now autogenetic, but the result of contagion.

There are numerous points of much clinical interest in connection with pyæmia which I must for the present pass by ; such, for instance, as the remarkable difference in the rapidity of development of the symptoms in different cases - the difference between the cases in which external abscesses form and those in which the abscesses are in the viscera. I must, however, before leaving the subject, remind you that the form of phlebitis which causes pyæmia is very different from another form which we not unfrequently witness in connection with varices, in which fibrinous plugging takes place. In these latter, there is no danger of softening or decomposition of the plug, and scarcely any as to its mechanical detachment. The plug which results from traumatic phlebitis is very different ; it is much softer, and it is loaded with cell-elements in addition to the fibrine. It is also attended not unfrequently by an almost gangrenous condition of the coats of the vein.

I have next to try to give you some idea of the class of cases to which we give the name Septicæmia or blood-poisoning. In these, the patient often dies very quickly, say within a few days of the injury, and long before phlebitic pyæmia could run its course. A rigor usually ushers in the attack, but it is not repeated, as in pyæmia; and, at the necropsy, congestions of the various viscera are found, but there are no isolated abscesses. Thus we may infer that this condition is not consequent upon phlebitis, or, at any rate, not upon the detachment of embola. This inference is supported by the fact that these cases do not follow operations on the veins or bones at all specially, but occur mainly after injuries to soft parts, or as the result of gangrenous inflam. mation of them. The phenomena of a severe case of septicæmia would suggest the inference that all the blood had passed into a state bordering on decomposition; and nothing is more remarkable than the extreme rapidity with which putrefaction sets in after death. It is sometimes very difficult to tell whether the symptoms during life are due to blood-poisoning or to nervous shock; indeed, it is quite an open question, whether nerve-shock does not take a large share in the production of the blood-changes which we name septicæmia. An elderly gentleman fell and dislocated his thumb. It was reduced; but the next day he was sick and shivery; and on the next day he was very ill, and had a scarlet efflorescence on his chest and abdomen. His thumb became gangrenous, and he died on the fifth day. Was this bloodpoisoning, or merely nervous shock from the commencing gangrene? In another case, $I$ amputated through the leg in a case in which the femoral artery was obliterated. Gangrene of the stump set in, and extended rapidly to the knee. The man was sick, delirious, and with a pulse at $\mathbf{r} 60$. Was he suffering from blood-poisoning as the result of absorption from the gangrenous part, or was he merely the subject of nervous shock? I amputated again high up in the thigh; his symptoms at once subsided, and he recovered. The event seemed to prove that, after all, it was nothing but shock; yet, had I left the limb on, no doubt he would have rapidly died, with the usual phenomena of "septicæmia." Thus we may admit that there is some difficulty in rightly interpreting the cases in which violent constitutional disturbance occurs in connexion with advancing gangrene, or severe inflammation threatening it. These supply us with a considerable proportion of the cases of so-called septicæmia. Almost always, indeed, the local inflammation runs high. Of course, with gangrene, or with very violent inflammation, it is 
always possible that the fluids may be taken up from the dying parts, which may act as blood-poisons ; but, on the other hand, as we have seen, it is possible to explain the symptoms in another way.

The cases in which occasionally surgeons or students die rapidly after dissection-pricks or wounds received in operating, are good examples of what is meant by septicæmia, as distinct from pyæmia ; and I may remark, that not unfrequer.tly there is evidence of violent implication of the lymphatic system. Now and then, but I think only rarely, a patient dies of septicæmia after an operation. In this disease, the convalescence may, in case of recovery, be protracted; but the outbreak of the symptoms is never long delayed, and in this respect the malady contrasts strongly with what is the case in pyæmia.

What the precise nature of the deleterious materies in septicæmia may be, whether living or dead, we know not; but this we assert, in contradistinction to pyæmia, that it is fluid, and has no tendency to cause embolic abscesses. Although it is not unfrequently seen after wounds which might be supposed to be possibly contaminated by some decomposing animal material (dissection-wounds, etc), yet we must clearly understand that it may originate where nothing of the kind was possible, as, for instance, after pricks with sewing-needles and the like. As to any predisposing condition of the general health, we can assert nothing. It is sometimes even imagined that what is called septicæmia may occur without any wound at all, but simply by cutaneous or pulmonary absorption. Thus you will see that the maladies grouped under this name differ considerably amongst themselves, and that their exact nature is far from being as yet well understood. From this, the vaguest group of our hospital plagues, I now turn your attention to the most definite.

The malady to which we give the name of Hospital Gangrene is one easily diagnosed, and in the large majority of cases its cause can be assigned with confidence. It prevails, when it does nccur, as an epidemic ; and, beyond all reasonable doubt, the cause of its spread is contagion. That the secretions from a sore in a state of phagedænic inflammation will breed phagedæna, as well in the subject of the disease as in others, is, I hold, most certain. It is by acting un this item of knowledge that we seek to cure the disease; and we must work in the same direction, if we wish to prevent it.

There are, I expect, but few present who have ever seen an example of hospital gangrene. It is not a malady which occurs sporadically ; we have no single cases now and then on which to demonstrate its characters; and I am thankful to say we have, in this hospital, had no epidemic for many years. I recollect three epidemics of it, two of them in this hospital. It is a horrible plague, and, on the last occasion, it spread so extensively, and persisted for so long, that we were obliged to desist from operating, excepting in the most urgent cases. Wound after wound was attacked, and in paiients of the most various states of health. At length, after having been with us, I think, nearly a year, it ceased, and we have not, so far as I know, had a case since. Cases of phagedæna, in connection with syphilis, we have had plenty of; but in no case have wounds in non-syphilitic persons been attacked, and it now never for a moment enters into our calculations of the risks of an operation. The hospital has been more crowded than ever; our cases have been of the same kind as formerly, and drawn from the same classes; but of hospital gangrene, we see nothing. I attribute this immunity to our care in isolating all venereal examples of the disease. In connection with this statement, let me repeat what I have often before said, that I cannot help suspecting that hespital gangrene usually starts from syphilitic cases. We have abundant, almost every-day proof that the syphilitic inflammation can originate it. It may attack the primary sore, the secondary eruption, or the tertiary ulcer. Without going to the length of denying that it may arise from other causes, I will simply say that $I$ have never seen examples of its autogenesis under other conditions. To this, I may add that the last epidemic of it in this hospital began in a corner of Mellish ward, close to the bed of a young man who had a phagedænic syphilitic sore. During the last few years, I have often stated my opinion on this point to you ; I have even gone so far as to suggest that syphilis may, for practical purposes, be considered the parent of phagedana. You may judge of the extreme interest with which, about a year ago, in conversation with Mr. Pollock of St. George's Hospital, I learnt that he entertained the same opinion as to the source of origin of the last epidemic of gangrene from which they suffered there. Mr. Pollock, without knowing my belief, told me that he had always suspected that it started from a syphilitic case which had been admitted into an open surgical ward.* Without being able to state that any observations have as yet been made of the disease, as met with

* I have Nr. Pollock's permission to give publicity to this statement. I may take advantage of this note to state also, that the term Hospital Plagues, which heads my lecture, is not original, but is one amongst the many singularly telling epithets which we owe to my former teacher Sir James Paget For the term autogenesis, $1 n$ the sense in which I have used it, I think I am indebted to Dr. Barnes. in army hospitals, which support my opinion, I believe I may suggest that there are none which confute it, and that the conditions of these hospitals are usually such as to make it quite possible. Nothing is more certain than that overcrowding, neglect of dressing, and a bad dietary are usually quite inadequate to the genesis of hospital gangrene. If once began, they all, as we may imagine, facilitate its spread, but something else is required to start it. I have suggested a possibility as to what that originating influence is, and it will be for future observation to con. firm or refute the conjecture.

And now, gentlemen, I must conclude this very fragmentary lecture by an attempt to sum up my conclusions. I have tried to insist that, in investigating the hospital plagues, we must keep clearly in mind two things-the causes of their origin (autogenesis) and the causes of their spread. Respecting one of the four, septicamia, my suggestion has been that it rarely spreads as an epidemic, and that most of its examples are, in a certain sense, autogenetic. It may occur almost as easily out of hospitals as in them. With the other three, the case stands, how. ever, very differently, since all of them may spread by contagion, and may become prevalent as genuine plagues in institutions where surgical cases are congregated. Pyæmia may arise by autogenesis whenever a vein inflames or is injured, or whenever a bone is contused, or when decomposing matter is left in contact with the uterine sinuses. It is common, and will always be so, in spite of all our precautions, both in hospitals and out of hospitals, in association with such causes as these. It may spread as an epidemic, if patients, with wounded veins, or broken bones, or torn uterine sinuses, be exposed to the contagion of erysipelas, or to the influence of certain other animal poisons. By preventing such risk, we may prevent pyæmia from becoming prevalent beyond a certain point. There is no hope of exterminating it, and there is, further, no reason to think that it is in any special way connected with the health of the patient, the food which he eats, or the air which he breathes. Erysipelas is very easily produced de novo, and also very easily spreads by contagion. In preventing both its autogenesis and its spread, the surgeon has much power and much responsibility. He must see that his patients' wounds are kept as healthy as possible ; that their secretions are not allowed to accumulate, and they are not exposed to the influence of draughts. Probably, numberless cases originate in hospitals in connection with exposure to open windows, which would be avoided in private houses. But the surgeon's main duty is to prevent its spreading by contagion, by scrupulously sending every case of erysipelas out of the ward, and by insisting that his dressers, nurses, etc., avoid, as far as possible, all risks of transference of secretions. As seldom as possible should his hands be allowed to touch an erysipelatous surface, and the most careful precau. tions, as regards washing, should always follow. If engaged in operating practice or in obstetrics, he should, as far as practicable, decline to see erysipelas cases. In reference to hospital gangrene, we have seen that its autogenesis is common in connection with syphilis, and very rare under other conditions, and that our duty, in order to prevent it, is to exclude syphilitic phagedæna from surgical wards.

Briefly, then, as regards the prevention of the epidemic spread of these pests, I have to beg you to believe in CoNTAGION, and in nothing else. Give your patients good food and good air; they will be the better for what you can do in these directions; but, if by attention either to diet or to ventilation, you hope to prevent a single case of erysipelas, pyæmia, or hospital gangrene, you are leaning on a reed. There is but one means by which this may be done, by which your wards may be kept healthy and your patients saved, and that is the prompt and systematic isolation of all contagious cases.

JACOBI'S FOOD FOR INFANTs. - Dr. J. D. Nourse writes in the Ohio Clinic, that a lady in his practice having lost three children within six months of their births from chronic diarrhœa as a consequence of artificial lactation, he urged her to try the food recommended by Dr. Jacobi of New York, in the case of an infant three months old, who was having from twelve to fifteen discharges every twenty-four hours as the result of artificial feeding, and to all appearance would die. The mother had a dread of diluted milk in any form, and in this case, but without success, was using cracker-water, diluted cream, etc. She gave the food as directed ; and, after two months' use, the child was growing and hearty, without diarrhœa. Dr. Nourse has recommended it in several other cases, and is confident it is the best artificial food that can be used. It is prepared as follows. Crack a teaspoonful of barley in a common coffee-mill, then boil it fifteen minutes in a gill of water, adding a pinch of salt. Then strain, and, for a young child, add half as much cow's milk as you have barley-water, and, whilst tepid, nurse from a nursing bottle. Sweeten lightly with sugar. If the bowels be costive, use oatmeal in place of barley. Keep the bottle clean. 